\title{
Lernen mit digitalen Lernressourcen am Beispiel von Khan Academy
}

\author{
Joseph Ferdinand, Sandra Engler, Christian Fischer
}

University of Tübingen, Germany

Pre-print for:

Ferdinand, J., Engler, S., \& Fischer, C. (2020). Lernen mit digitalen Lernressourcen: Beispiel Khan Academy [Learning with online materials: The example of Khan Academy]. Schulmanagement, 5, 24-27.

Please note that this pre-print represents the manuscript version submitted for review and prior to changes from the peer review and copy-editing processes. The final peer-reviewed version appeared in Schulmanagement. The article has been published and here is the link. 


\title{
Lernen mit digitalen Lernressourcen am Beispiel von Khan Academy
}

\author{
Joseph Ferdinand, Sandra Engler und Christian Fischer \\ University of Tübingen
}

Abstract: Weltweit sind, nicht zuletzt inspiriert durch YouTube und Co, viele digitale Lernplattformen entstanden, um Schüler*innen in ihren Lernprozessen zu unterstützen. Eine solche Lernplattform ist Khan Academy. Auf Khan Academy können Nutzer Inhalte vornehmlich zu naturwissenschaftlichen Themen mittels kurzer Erklärvideos und Übungsaufgaben angepasst an individuelle Lernstände und -geschwindigkeiten erarbeiten. Arbeitsweise und Lernfortschritt können von einem Tutorensystem unterstützt werden. Zudem gibt es weitreichende Monitoring-Funktionen für Lehrkräfte, die einen Einsatz der Anwendung als Ergänzung des Regelunterrichts ermöglichen. Erste empirische Studien deuten auf die Lernförderlichkeit im Einsatz von Khan Academy hin.

\section{Einführung}

In den letzten Jahrzehnten wurden immer häufiger digitale Medien im schulischen Unterricht eingesetzt (Wößmann et al, 2017; Eickelmann, 2018). Insbesondere aufgrund der aktuellen Corona-Pandemie wird die Notwendigkeit von digitalen Unterstützungsangeboten ersichtlich: viele Bundesländer in Deutschland haben auf die Situation schnell reagiert und verschiedene Plattformen für den Unterricht zu Hause zur Verfügung gestellt (Bitkom, 2020). Dennoch sind häufig verwendete Plattformen wie Moodle eher für die Verwaltung von Nutzern und Lerninhalten geeignet. Andere Lernplattformen sind mit spezifischeren pädagogischen Lernzielen konzipiert. Eine dieser Plattformen ist Khan Academy, welche mit Prinzipien des Adaptiven Lernens mit Gamification-Elementen arbeitet, um Lernenden zu unterstützen und zu motivieren (Klock et al., 2015; Tenório et al. 2018). 


\section{Die Lernplattform}

Khan Academy wurde im Jahr 2008 als Non-Profit Organisation von Salman Khan gegründet. Zuvor gab der gebürtige US-Amerikaner mit Hochschulabschlüssen in Elektrotechnik, Informatik, Mathematik, und Business Administration über viele Jahre sehr erfolgreich Nachhilfe. Zunächst nur seiner Verwandtschaft über die Doodle Notepad App. Als das Interesse an individueller Nachhilfe seine Kapazitäten überschritt, begann Salman Khan seine Erklärvideos auf einem eigens eingerichteten YouTube Kanal namens „Khan Academy“ anzubieten. Durch den großen Erfolg seiner Erklärvideos kündigte er im Jahr 2009 seinen Job als Finanzanalyst und widmet sich seitdem hauptberuflich der Weiterentwicklung seiner gleichnamigen Lernplattform. Deren Inhalte werden von einem großen Team aus Lehrkräften, Wissenschaftlern und Experten für Test-Erstellung und -Durchführung auf ihre fachliche und pädagogische Qualität überprüft. Khan Academy wird hauptsächlich in englischer Sprache betrieben, jedoch werden Aufgaben und Themenbereiche (vor allem im mathematischnaturwissenschaftlichen Bereich) aktuell in 59 Sprachen angeboten. Khan Academy kann dabei sowohl von Schüler*innen zum selbständigen Lernen verwendet als auch von Lehrkräften oder Eltern unterstützend eingesetzt werden.

\section{Erklärvideos als zentrale Methode der Wissensvermittlung}

Als zentrales Medium der Wissensvermittlung verwendet Khan Academy Erklärvideos mit der „Live-Scribble“ Methode. Dabei wird auf schwarzem Hintergrund innerhalb weniger Minuten ein thematisch abgegrenzter Sachverhalt erklärt und durch nachverfolgbare Notizen des Moderators ergänzend verständlich gemacht.

Motivationsfördernd soll dabei die eher personalisierte und wenig hierarchischen Art der Präsentation wirken, die Jugendliche auch aus ihrem Freizeitgebrauch kennen (Zander et al., 2018). Dies spiegelt sich auch in der verstärkten Nutzung von Erklärvideos auf Plattformen wie YouTube als Informationsquelle von Jugendlichen wider: Laut der Studie „Jugend, 
Information, Medien” zum Medienumgang 12- bis 19-Jähriger des Medienpädagogischen Forschungsverbund Südwest (MPFS) nutzen 20\% der Mädchen und 19\% der Jungen die Streaming-Plattform für Tutorials (MPFS, 2019), im Vergleich dazu waren es 2016 nur 10 bzw. 9\% (MPFS, 2016).

Erklärvideos bieten Lernenden dabei Möglichkeiten der selbständigen Gestaltung von Lernprozessen indem Videos pausiert, wiederholt angesehen bzw. angehört und in Vortragsgeschwindigkeit beschleunigt oder verlangsamt werden können. Solche Steuerungsmöglichkeiten können kognitiver Überforderung und der Angst vor dem Verpassen wichtiger Informationen entgegenwirken (Merkt et al., 2011).

\section{Aufbau von Themen bei Khan Academy}

Ein Thema besteht aus mehreren Lerneinheiten, welche in zu erreichenden Fähigkeiten, die als „Skills“ bezeichnet werden, untergliedert sind. Jede der Lerneinheiten besteht aus einer festgelegten Anzahl Skills, die benötigt werden, um die Einheit bzw. das Lernthema zu meistern. Um das „Mastery-Level“ eines Skills zu erreichen bearbeiten Lernende verschiedene Lernaktivitäten. Für jede Lernaktivität kann dabei eine transparent festgelegte Anzahl an Punkten gesammelt werden. Lernende erhalten für das Erreichen festgelegter Lernfortschritte Belohnungen in Form von Abzeichen, die automatisch vergeben

und gesammelt werden. Sobald eine bestimmte Anzahl an Punkten einer Lerneinheit erreicht wurde, können mit einer Abschlussprüfung (die sogenannte „Kurs-Challenge“) alle Wissensbereiche der Lerneinheit abgeprüft werden. Dabei sind Lern- und Bearbeitungsfortschritte aller verfügbarer Themen auf Khan Academy zu jeder Zeit von Lernenden, Lehrkräften oder Eltern einsehbar.

\section{Unterstützung für die Unterrichtsgestaltung}

Lehrkräfte können sich bei Khan Academy ein kostenfreies Lehrer-Konto einrichten und die verfügbaren Inhalte nach Bedarf individuell im Unterricht einsetzen. $\mathrm{Zu}$ den 
Themenfeldern bestehen vollständig ausgearbeitete „Unterrichtsstunden“ (z.B. Mathematik, Klasse 8; Abbildung 1), wobei jedoch eine Passung an Lehrpläne von Lehrkräften überprüft werden sollte. Daher ist es eher zu empfehlen bedarfsgemäß für die individuelle Unterrichtssituation aus den angebotenen Materialen zu wählen und beispielweise ausgewählte Lerneinheiten als Vor- oder Nachbereitung des Unterrichts oder auch als Hausaufgabe einzusetzen. Eine alleinige Nutzung von gesamten Unterrichtseinheiten wäre beispielsweise denkbar als Einführung in neue Themenbereiche, als binnendifferenzierte Wiederholung für leistungsschwächere Schüler*innen (oder als Vorgriff auf zukünftige Themen für leistungsstarke Schüler*innen ), sowie als temporär begrenzter „Ersatz“ im Krankheitsfall, um Unterrichtsausfall entgegen zu wirken.

[Abbildung 1 ungefähr hier]

Ein wesentlicher Bestandteil zur Unterstützung von Lehrkräften im Unterricht ist das gut ausgearbeitete Monitoringsystem, das individuelle Lernfortschritte jedes Lernenden und der Lerngruppen aufzeigt. Lehrkräften können beispielsweise übersichtlich erkennen, welche Aufgaben (und mit welchem Erfolg) bereits bearbeitet wurden und welche Videos angesehen wurden. Durch die Übersicht allen Lernfortschritts kann Lehrkräften ersichtlich werden, wo Schwierigkeiten bestehen, um eine individuelle Förderung zu veranlassen (siehe

Abbildung 2). Ebenso können besonders leistungsstarken Schüler*innen n mit wenigen Klicks weiterführende Lerneinheiten zugeteilt werden.

[Abbildung 2 ungefähr hier]

\section{Einstieg in der Verwendung von Khan Academy für Lehrkräfte}

Khan Academy bietet Lehrkräften sehr ausführliche Online-Anleitungen in Form von Lerneinheiten für Lehrkräfte, durch welche die Bedienung der Plattform erläutert wird.

Weiterhin gibt es eine Online Teacher-Community und einen Online-Support mit Artikeln zur Beantwortung der häufigsten Anfragen und der Möglichkeit des E-Mail-Kontakts für 
weiterreichende Fragen. Nach Erstellen eines kostenfreien Lehrerkontos über die Startseite (https://de.khanacademy.org) kann auf das sogenannte Lehrer-Dashboard zugegriffen werden. Dabei können Lehrkräfte beliebig viele Kurse für beliebig viele Lerngruppen mit unterschiedlichen Themen erstellen. Es ist daher möglich Themen von einer ganzen Jahrgangsstufe, einer einzelnen Klasse oder ausgewählten Schüler*innen bearbeiten zu lassen.

Kurse können aus Google Classroom importiert oder im Lehrer-Dashboard neu erstellt werden. Je nach gewähltem Themenbereich stehen bereits Unterthemen mit Erklärvideos, Übungsaufgaben und Artikeln bereit. Über die Funktion "Ziel erstellen” können ein oder mehrere Ziele für einzelne oder alle Lernende festgelegt werden. Mittels der Funktion „Arbeitsaufträge - Zuweisen“ können Schüler*innen Aufgaben wie dem Ansehen von Videos oder Bearbeiten von Übungen und Tests aufgetragen werden.

Lerngruppen können ebenfalls entweder von Google Classroom importiert oder neu erstellt werden. Bei Neuerstellung können sich Schüler*innen über kurspezifische Codes Lerngruppe hinzufügen oder von der Lehrkraft initiierte E-Mail-Einladungen über Khan Academy erhalten. Alternativ können Lehrkräfte Schüleraccounts anlegen. Für die Einrichtung des Accounts von Kindern unter 13 Jahren wird die Zustimmung der Eltern benötigen, da die Plattform als Partner des „Student Privacy Pledge“ (https://studentprivacypledge.org/privacy-pledge) Minderjähriger besonders schützen möchte. Khan Academy bietet für den Unterrichtseinsatz eine vorgefertigte deutschsprachige Elterninformation an. Zur Einhaltung der Datenschutz- und Privatsphäre-Regelungen für die Schüler*innen verpflichtet sich jedoch die Lehrkraft durch die Zustimmung zur AGB bei Kurserstellung.

Das Lehrer-Dashboard zu Lerngruppen umfasst dabei weitreichen Funktionen zum Kursmanagement. Lehrkräfte können für alle Schüler*innen individuelle Aktivitätenprotokolle (Lern- und Übungsminuten sowie Skills eines Lernenden), sowie einen 
Überblick über den Status aller Arbeitsaufträge erhalten. Die Niveaus der zu erarbeitenden Skills werden dabei graphisch als fortlaufende Balken in vier Abstufungen angezeigt (Versucht, Kenner, Profi, Experte; Abbildung 3). In der Kurs-Mastery Ansicht werden Fortschritte der Lernenden differenzierter verdeutlicht: Die maximal möglichen sowie erreichten Punktzahlen werden in Quintilen pro Lerneinheit und in Prozent die Gesamtleistung dargestellt. Zudem kann eine tabellarische Information des Bearbeitungsstatus der Arbeitsaufträge aller Lernenden, sowie die am häufigsten falsch beantworteten Fragen angezeigt werden. Dies kann sich zur Nachsteuerung und Wiederholung von Themen eignen. Weiterhin können Lehrkräfte wöchentliche E-MailBenachrichtigungen abonnieren, die Zusammenfassungen der Fortschritte der Lerngruppen beinhalten.

[Abbildung 3 ungefähr hier]

\section{Effektivität von Khan Academy}

Digitale Medien sollen in erster Linie Lernprozesse unterstützen, und keinesfalls als vollständiger Ersatz von traditioneller Lehre gesehen werden. Vor diesem Hintergrund stellen wir einige Studien vor, welche Khan Academy hinsichtlich Akzeptanz, Beschäftigungsdauer mit Lerninhalten, und Lernleistung untersuchen.

\section{Akzeptanz}

Die Akzeptanz einer Plattform als Lernmedium stellt eine Grundvoraussetzung für deren effektive Nutzung dar (Holden \& Karsh, 2010; Davis, 2011). Die Akzeptanz hängt dabei jedoch von der Benutzerfreundlichkeit und der wahrgenommenen Nützlichkeit ab (Davis, 1989; Venkatesh \& Davis, 2000). Laut einer aktuellen Studie berichteten Lernende, dass Khan Academy sowohl benutzerfreundlich als auch hilfreich zum Erreichen eigener Lernziele ist (Arnavut et. al, 2019). Insbesondere haben Lernende, welche die Nutzung der 
Plattform als herausfordernd eingestuft haben, die spielerischen Elemente (z.B. Abzeichen und Punkte) als lernhilfreich empfunden (Arnavut et. al, 2019).

\section{Beschäftigungsdauer mit Lerninhalten}

Um Lehr-Lernprozesse mit Khan Academy besser zu verstehen haben Light und Pierson (2014) acht Lehrpersonen, sechs Schulverwalter und 32 Schüler*innen interviewt, sowie 25 Mathematikstunden beobachtet. Es zeigte sich, dass die Schüler*innen, die mit Khan Academy gelernt haben, sich länger mit mathematischen Aufgaben beschäftigt haben als Schüler*innen im traditionellen Unterricht. Dies kann auf die spielerischen Elemente der Lernplattform, den sofortigen Rückmeldungen bei Aufgabenbearbeitung, sowie den Möglichkeiten zur Wiederholung von Aufgabentypen zurückgeführt werden. Die Interviews stellten dabei heraus, dass Schüler*innen insbesondere vom Erwerb von Abzeichen und der Punktestrukturen motiviert wurden. Dieses gesteigerte Engagement könnte sich dann positiv auf den Kompetenzerwerb auswirken.

\section{Lernleistung}

Eine großangelegte Studie untersuchte in 20 verschiedenen Schulen wie sich Khan Academy auf die Mathematikleistung von Schüler*innen der 9. Klasse auswirkt (Weeraratne \& Chin, 2018). Schulen wurden dabei zufällig der Experimentalgruppe oder Kontrollgruppe zugeteilt. In der Experimentalgruppe wurden die Inhalte von 2-3 (von insgesamt 5) Unterrichtsstunden mit Khan Academy erlernt. Die restlichen Inhalte wurden im Präsenzunterricht vermittelt. In der Kontrollgruppe fand ausschließlich Präsenzunterricht statt. Es zeigte sich, dass Schüler*innen, die mit Khan Academy gelernt haben, bessere Leistungen erzielten als Schüler*innen, die ausschließlich mit klassischem Unterricht gelernt haben (Weeraratne \& Chin 2018).

Eine weitere Studie untersuchte den Einsatz von Khan Academy im „Flipped Classroom” Modell: Dieser pädagogische Ansatz kehrt die traditionelle Unterrichtssequenz 
um, sodass die Wissensvermittlung mittels Erklärvideos als „Hausaufgaben“ stattfindet, während Fragen, Anwendungen und Übungen im klassischen Unterrichtskontext durchgeführt werden (Bergmann \& Sams, 2012). Dabei zeigte Zengin (2017), dass der Einsatz von Khan Academy zu einer verbesserten Mathematikleistung führte. Weitere Studien verglichen verschieden Interventionen, wie beispielsweise „Accelerated Math“ (Renaissance Learning, 2020) und Khan Academy. In der Studie von Adams (2016) wurden dabei die Mathematikleistungen von 70 Schüler*innen der 5. Klasse verglichen. Zwar konnte kein Unterschied zwischen den Gruppen gefunden werden. Es zeigte sich jedoch eine moderate positive Korrelation zwischen der Zeit, die Schüler*innen in Khan Academy verbrachten und ihrer Mathematikleistung (Adams, 2016).

\section{Herausforderungen im Einsatz von Khan Academy}

Khan Academy erfährt oftmals Kritik aufgrund des zugrundeliegenden traditionellen transmissiven Modells der Wissensvermittlung und behavioristischen Lehr-Lernprozessen. Anstelle einer Lehrkraft an der Tafel wird die Wissensvermittlung mit Erklärvideos durchgeführt (Ani, 2013). Gleichfalls stehen beim Bearbeiten von Aufgaben repetitive Merkmale zum Fertigkeitserwerb im Vordergrund. Dies steht unter anderem im Gegensatz zu konstruktivistischen Modellen wie dem „Forschenden Lernen“ (z.B. Pedaste et al., 2015). Für eine Diskussion der Vor- und Nachteile dieser unterschiedlichen Arten der Wissensvermittlung sei beispielhaft auf Kirschner, Sweller und Clark (2006) sowie HmeloSilver, Duncan und Chinn (2007) verwiesen.

Weiterhin bietet Khan Academy aufgrund technischer Begrenzungen vor allem Lernangebote für mathematisch-naturwissenschaftliche Themen. Diese Inhaltsbereiche zeichnen sich insbesondere dadurch aus, dass notwendige Kompetenzen in kleinere aufeinander aufbauende Wissens- und Fertigkeitsteile unterteilt werden können, wodurch sie gut programmierbar sind. Sprach- und Schreibkompetenzen oder andere komplexe 
Themenbereiche, die sowohl analytische als auch kreative Fähigkeiten voraussetzen, sind jedoch weitaus schwieriger in einer solcher Lernplattform zu erwerben. Dies spiegelt sich darin wider, dass die meisten Angebote eher für den Erwerb von Basiswissen und Grundfertigkeiten angelegt sind, welche sich besser mit behavioristischen Lehrmethoden vermitteln lassen (Ani, 2013; Parslow, 2012). In diesem Zusammenhang zeigt eine Studie von Barman (2013), dass Khan Academy zwar effektiv zur Vermittlung einfacher Mathematikkonzepte ist, Lernende jedoch bei schwierigeren und komplexeren Mathematikkonzepten geringe Leistung aufweisen. Ebenso ist aus Sicht der pädagogischen Psychologie zu beachten, dass viele strukturelle Elemente von Khan Academy (z.B. Abzeichen, Punkte, etc.) eher auf die extrinsische Motivation der Lernenden abzielen. Es ist fraglich, inwieweit dies im schulischen Kontext auch zu erhöhter intrinsischer Motivation sowie zu gesteigertem Interesse an den jeweiligen Lernbereich führen kann. Ferner ist es notwendig, dass sich Lehrkräfte für den Einsatz von digitalen Plattformen aus- und fortgebildet werden, um mit diesen gezielt und lernförderlich die unterrichtliche Praxis zu unterstützen (Fischer \& Dershimer, 2016; Fischer et al., 2018). Schließlich sollte beim Einsatz von digitalen Medien wie Khan Academy auch berücksichtigt werden, dass Schüler*innen Zugang zur notwendigen Infrastruktur besitzen. Obwohl in Deutschland flächendeckender Internetzugang möglich ist, beeinflusst die soziale Herkunft von Schüler*innen deren tatsächliche und effektive Nutzung (Eickelmann et al. 2019).

\section{Fazit}

Grundsätzlich lässt sich festhalten, dass Khan Academy den klassischen Unterricht auch in der aktuellen Corona Pandemie - durch kostenfreie, digitale Unterstützungsangebote bereichern könnte. Erste Studien deuten darauf hin, dass Khan Academy einen positiven Einfluss auf die Dauer und die Intensität der Beschäftigung von Lernenden mit den Lerninhalten, sowie deren Lernleistung haben kann. Die Robustheit dieser Ergebnisse, sowie 
Übertragbarkeit auf verschiedene Kontexte, sollten jedoch in weiteren empirischen Studien belegt werden. Weiterhin sollte berücksichtigt werden, dass Khan Academy in erster Linie zur Unterstützung des mathematisch-naturwissenschaftlichen Unterrichts Angebote bietet. Dennoch sollten die Vorteile im ergänzenden Einsatz digitaler Lerngelegenheiten wie Khan Academy bei der Gestaltung modernen Unterrichts und den aktuellen digitalen Unterstützungsangeboten der Bundesländer in Betracht gezogen werden, auch wenn sie traditionelle Unterrichtangebote in keinem Fall vollständig ersetzen können oder sollten. 


\section{References}

Adams, D. L. (2016). Action research on the effectiveness of Khan Academy as a tier I intervention (Doctoral dissertation, Miami University).

Ani, K. K. (2013). Khan Academy: The hype and the reality. The Education Digest, 78(6), 23.

Arnavut, A., Bicen, H., \& Nuri, C. (2019). Students' Approaches to Massive Open Online Courses: The Case of Khan Academy. BRAIN. Broad Research in Artificial Intelligence and Neuroscience, 10(1), 82-90.

Barman, N. (2013). An evaluation of the effectiveness of the Khan Academy videos for teaching mathematics at Menzi High School. Independent Study Project (ISP) Collection. Retrieved from digitalcollections.sit.edu/isp_collection/1501

Bergmann, J., \& Sams, A. (2012). Flip your classroom: Reach every student in every class every day. International Society for Technology in Education.

Bitkom (2020). Digitale Lernangebote der Bundesländer in Zeiten von Corona. Retrieved August, 2020, from https://www.bitkom.org/Themen/Digitale-Unterstuetzung-inZeiten-von-Corona/Digitale-Lernangebote-der-Bundeslaender

Davis, F.D. (1989). Perceived usefulness, perceived ease of use and user acceptance of information technology. MIS Quarterly, 13 (3).

Davis, F. D. (2011). Foreword in technology acceptance in education: Research and issues. Rotterdam, The Netherlands: Sense Publishers.

Eickelmann, B. (2018). Digitalisierung in der schulischen Bildung - Entwicklungen, Befunde und Perspektiven für die Schulentwicklung und die Bildungsforschung. In N. McElvany, F. Schwabe, W. Bos \& H.G. Holtappels (Hrsg.), Digitalisierung in der schulischen Bildung. Chancen und Herausforderungen (S. 11-25). Münster: Waxmann.

Eickelmann, B., Bos, W., \& Labusch, A. (2019). Die Studie ICILs 2018 im Überblick. Zentrale Ergebnisse und mögliche Entwicklungsperspektiven. In ICILS 2018 Deutschland. Computer- und informationsbezogene Kompetenzen von Schüler*innen $n$ im zweiten internationalen Vergleich und Kompetenzen im Bereich Computational Thinking (pp. 7-31).

Fischer, C. \& Dershimer, R. C. (2016). Preparing teachers to use educational games, virtual experiments, and interactive sciencex simulations to engage students in the practices of science. In L.-J. Thoms \& R. Girwidz (Eds.), Proceedings of the 20th International Conference on Multimedia in Physics Teaching and Learning (pp. 263-270).

Mulhouse, France: European Physical Society. https://doi.org/10.31219/osf.io/yhkc3

Fischer, C., Fishman, B., Dede, C., Eisenkraft, E., Foster, B., Frumin, K., Lawrenz, F., Levy, A., \& McCoy, A. (2018). Investigating relationships between school context, teacher professional development, teaching practices, and student achievement in response to a nationwide science reform. Teaching and Teacher Education, 72, 107-121. https://doi.org/10.1016/j.tate.2018.02.011

Hmelo-Silver, C. E., Duncan, R. G., \& Chinn, C. A. (2007). Scaffolding and achievement in problem-based and inquiry learning: a response to Kirschner, Sweller, and Clark (2006). Educational Psychologist, 42(2), 99-107.

Holden, R. J., \& Karsh, B. T. (2010). The technology acceptance model: its past and its future in health care. Journal of Biomedical Informatics, 43(1), 159-172.

Khan Academy. Abgerufen am 03.08.2020, von https://de.khanacademy.org/

Kirschner, P. A., Sweller, J., \& Clark, R. E. (2006). Why minimal guidance during instruction does not work: An analysis of the failure of constructivist, discovery, problem-based, experiential, and inquiry-based teaching. Educational Psychologist, 41(2), 75-86. 
Klock, A. C. T., Da Cunha, L. F., de Carvalho, M. F., Rosa, B. E., Anton, A. J., \& Gasparini, I. (2015, August). Gamification in e-learning systems: A conceptual model to engage students and its application in an adaptive e-learning system. In International Conference on Learning and Collaboration Technologies (pp. 595-607). Springer, Cham.

Light, D., \& Pierson, E. (2014). Increasing student engagement in math: The use of Khan Academy in Chilean classrooms. International Journal of Education and Development using ICT, 10(2), 103-119.

Medienpädagogischer Forschungsverbund Südwest. (Hrsg.). (2016). JIM-Studie 2016. Jugend, Information, (Multi-) Media. Basisuntersuchung zum Medienumgang 12- bis 19-Jähriger.

https://www.mpfs.de/fileadmin/files/Studien/JIM/2016/JIM_Studie_2016.pdf. Zugegriffen am 07.08.2020

Medienpädagogischer Forschungsverbund Südwest. (Hrsg.). (2019). JIM-Studie 2019. Jugend, Information, Medien. Basisuntersuchung zum Medienumgang 12- bis 19Jähriger. https://www.mpfs.de/fileadmin/files/Studien/JIM/2019/JIM_2019.pdf. Zugegriffen am 07.08.2020

Merkt, M., Weigand, S., Heier, A. \& Schwan, S. (2011). Learning with videos vs. learning with print: The role of interactive features. Learning and Instruction, 21 (6), pp. 687704. https://doi.org/10.1016/j.learninstruc.2011.03.004

Parslow, G. R. (2012). Commentary: The Khan Academy and the day-night flipped classroom. Biochemistry and Molecular Biology Education, 40(5), 337-338.

Pedaste, M., Mäeots, M., Siiman, L. A., De Jong, T., Van Riesen, S. A., Kamp, E. T., ... \& Tsourlidaki, E. (2015). Phases of inquiry-based learning: Definitions and the inquiry cycle. Educational Research Review, 14, 47-61.

Renaissance Learning. (2016). Reasearch. Renaissance Learning. Retrieved from: http://www.renaissance.com/Resources/Research

Tenório, M. M., Lopes, R. P., Góis, L. A. D., \& Junior, G. D. S. (2018). Influence of gamification on Khan Academy in Brazilian high school. PUPIL: International Journal of Teaching, Education and Learning, 2(2), 51-65.

Venkatesh, V., \& Davis, F. D. (2000). A theoretical extension of the technology acceptance model: Four longitudinal field studies. Management science, 46(2), 186-204.

Weeraratne, B., \& Chin, B. (2018). Can Khan Academy e-Learning Video Tutorials Improve Mathematics Achievement in Sri Lanka? International Journal of Education and Development using Information and Communication Technology, 14(3), 93-112.

Wößmann, L., Lergetporer, P., Grewenig, E., Kugler, F., \& Werner, K. (2017). Fürchten sich die Deutschen vor der Digitalisierung? Ergebnisse des ifo Bildungsbarometers 2017. ifo Schnelldienst, 70(17), 17-38.

Zengin, Y. (2017). Investigating the use of the Khan Academy and mathematics software with a flipped classroom approach in mathematics teaching. Journal of Educational Technology \& Society, 20(2), 89-100. 


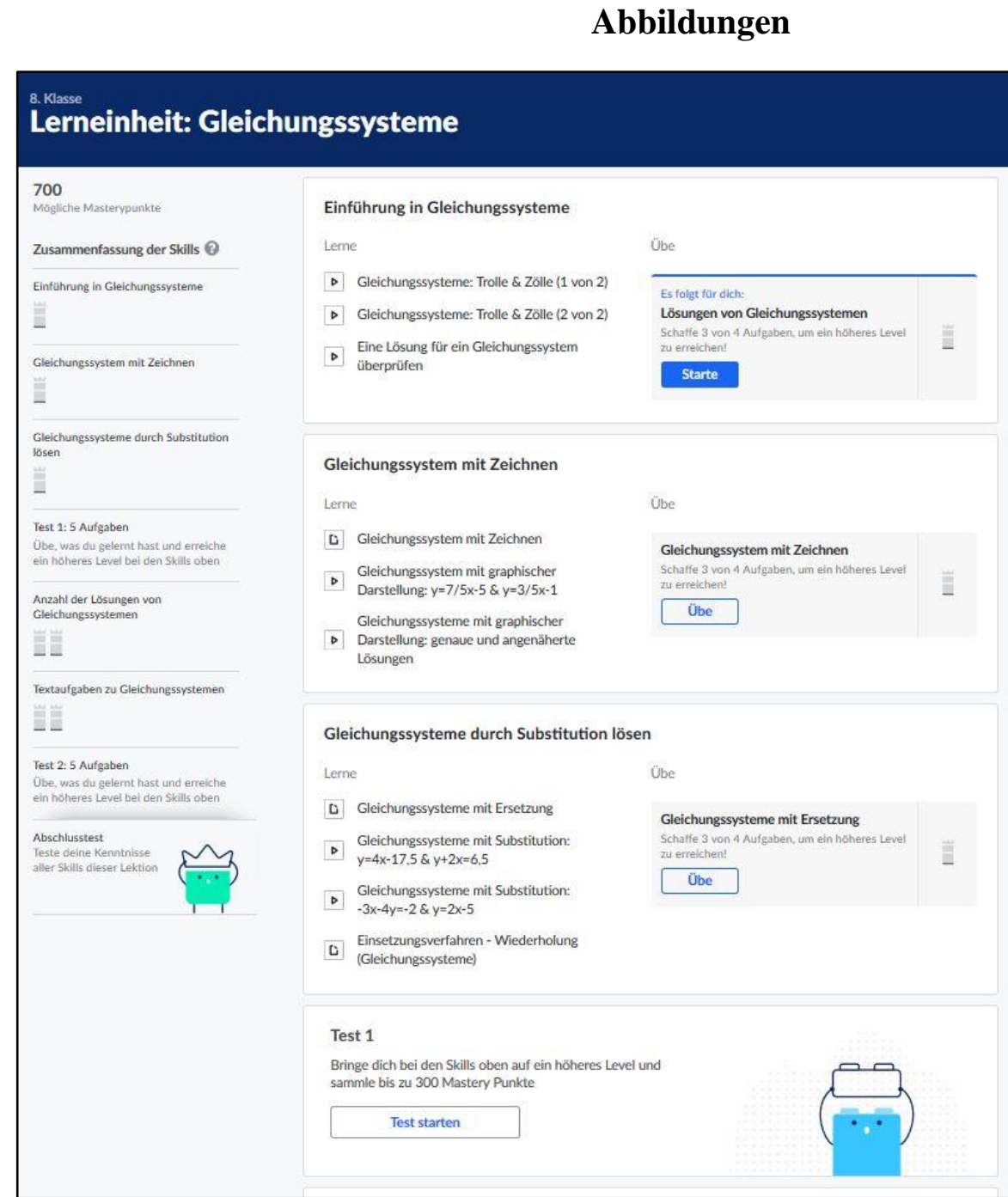

Abbildung 1. Lerneinheit im Fachbereich Mathematik zum Thema „Gleichungssysteme“.

\section{Ergebnisse der Arbeitsaufträge}

Hier ist, wie deine Schüler bei den Themen stehen, die du ihnen zugewiesen hast. Du kannst den Namen eines Arbeitsauftrag anklicken, um detaillierte Berichte zu erhalten.

Allzeit

\begin{tabular}{|c|c|c|c|c|c|c|c|c|c|c|c|}
\hline SCHOLLER/IN & 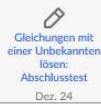 & 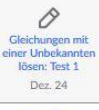 & $\begin{array}{c}\Delta \\
\text { Einfuhrung in } \\
\text { Gleichungen mit } \\
\text { Variablen auf } \\
\text { beiden Seten } \\
\text { Dez. } 24\end{array}$ & 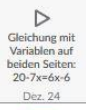 & $\begin{array}{c}8 \\
\text { Gleichungen mitt } \\
\text { Variaboln aut } \\
\text { beiden Seiten } \\
\text { Dez. } 24\end{array}$ & 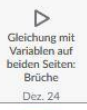 & 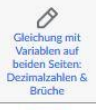 & $\begin{array}{c}D \\
\text { Gleichungen mit } \\
\text { Klammern } \\
\text { Dez. } 24\end{array}$ & 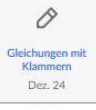 & $\begin{array}{l}\Delta \\
\text { Gleichungen mit } \\
\text { Klammer: } \\
\text { Dezimalzahlen \& } \\
\text { Bruche } \\
\text { Dez. } 24\end{array}$ & $\begin{array}{l}\text { Mehrschitige } \\
\text { cleichungen. } \\
\text { Wiedertholung } \\
\text { Dez. } 24\end{array}$ \\
\hline Beispiel Schüler 1 & & 60 & -1 & $\checkmark$ & 100 & $\checkmark$ & 0 & -2 & 100 & & $\checkmark$ \\
\hline Beispiel Schüler 2 & & 80 & - & - & 100 & $\checkmark$ & 50 & -2 & 100 & - & - \\
\hline Beispiel Schülerin 1 & - & 80 & -1 & - & 100 & $\checkmark$ & 75 & $\checkmark$ & 100 & - & - \\
\hline Beispiel Schülerin 2 & 78 & 100 & 2 & - & 100 & $\checkmark$ & 100 & - & 100 & 100 & - \\
\hline
\end{tabular}

Abbildung 2. Übersicht der Lernfortschritte einer Beispielklasse. 


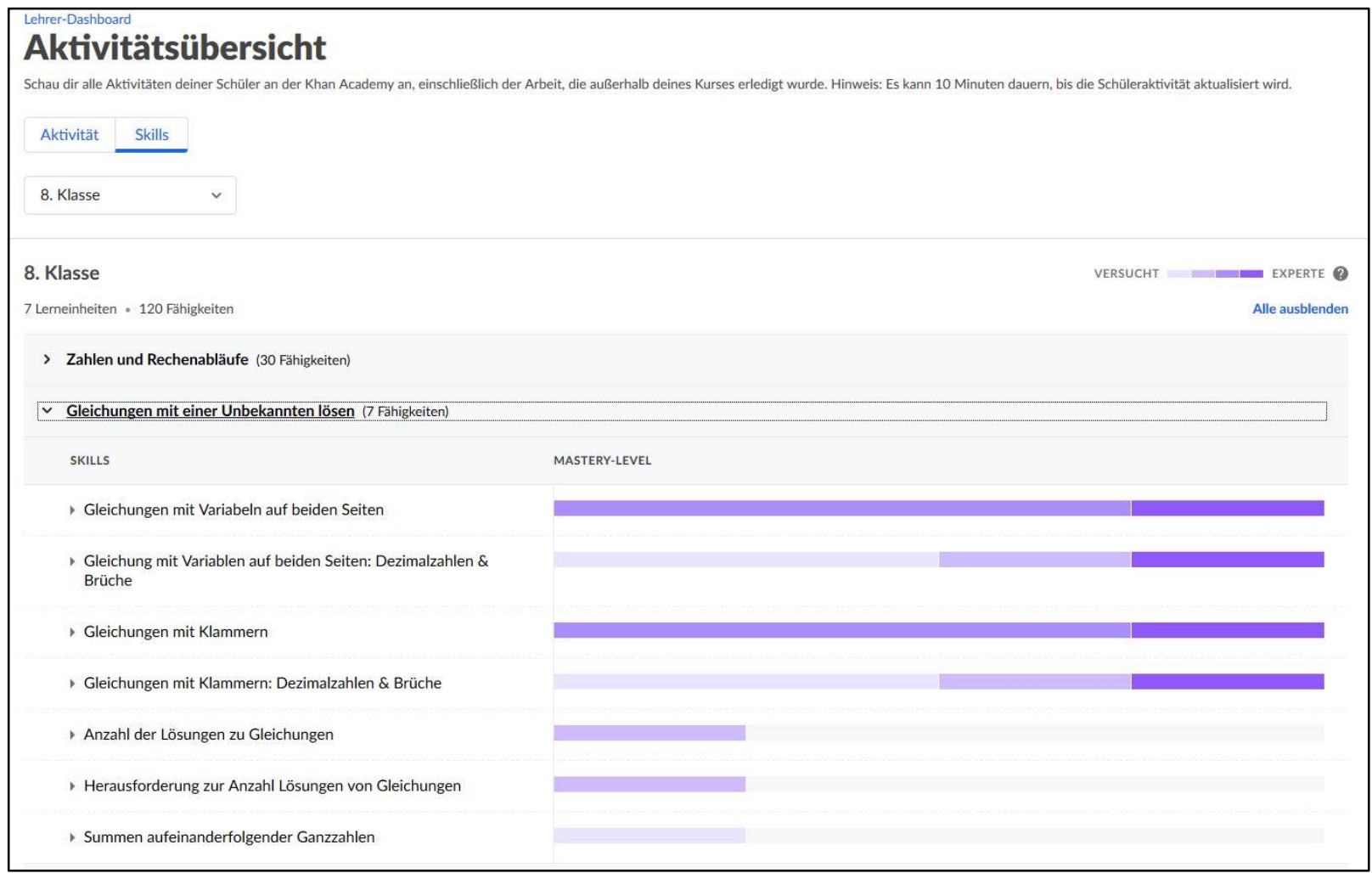

Abbildung 3. Übersicht der Skill-Level eines Mathematikkurses im Lehrer-Dashboard. 